\title{
Bax activation and mitochondrial insertion during apoptosis
}

\author{
Lisenn Lalier • Pierre-François Cartron • \\ Philippe Juin • Svetlana Nedelkina • Stephen Manon • \\ Burkhart Bechinger · François M. Vallette
}

Published online: 15 February 2007

(C) Springer Science + Business Media, LLC 2007

\begin{abstract}
The mitochondrial apoptotic pathway is a highly regulated biological mechanism which determines cell fate. It is defined as a cascade of events, going from an apoptotic stimulus to the MOM permeabilization, resulting in the activation of the so-called executive phase. This pathway is very often altered in cancer cells.
\end{abstract}

L. Lalier · P.-F. Cartron · P. Juin · F. M. Vallette

INSERM U601,

F-44000 Nantes, France

L. Lalier · P.-F. Cartron · P. Juin · F. M. Vallette

Université de Nantes, Faculté de Médecine,

9 Quai Moncousu,

F-44035 Nantes., Cedex 01, France

S. Nedelkina $\cdot$ B. Bechinger

CNRS LC3-UMR7177,

F-67070 Strasbourg, France

S. Nedelkina $\cdot$ B. Bechinger

Université Louis Pasteur, Institut/Faculté de Chimie,

4, rue Blaise Pascal,

F-67070 Strasbourg, France

\section{S. Manon}

Institut de Biochimie et Génétique,

Cellulaires, UMR5095 CNRS,

33077 Bordeaux, Cedex, France

\section{S. Manon}

Université de Bordeaux 2,

1 rue Camille Saint Saens,

33077 Bordeaux, Cedex, France

F. M. Vallette $(\bowtie)$

INSERM U601, Université de Nantes, Faculté de Médecine,

9 Quai Moncousu,

F-44035 Nantes. Cedex 01, France

e-mail: Francois.vallette@univ-nantes.fr
The mitochondrial permeabilization is under the control of the Bcl-2 family of proteins (pBcls). These proteins share one to four homology domains (designed BH1-4) with Bcl2 , and are susceptible of homo- and/or hetero-dimerization. In spite of a poor amino-acid sequence homology, these proteins exhibit very similar tertiary structures. Strikingly, while some of these proteins are anti-apoptotic, the others are proapoptotic. Pro-apoptotic proteins are further divided in two sub-classes: multi-domains proteins, among which Bax and Bak, which exhibit BH1-3 domains, and BH3-only proteins (or BOPs). Schematically, BOPs and anti-apoptotic proteins antagonistically regulate the activation of the multi-domain proteins Bax and Bak and their oligomerization in the MOM, the latter process being responsible for the apoptotic mitochondrial permeabilization.

Considering the critical role of Bax in cancer cells apoptosis, we focus in this review on the molecular events of Bax activation through its interaction with the other proteins from the Bcl-2 family. The mechanism by which Bax triggers the MOM permeabilization once activated will be discussed in some other reviews in this special issue.

Keywords Apoptosis $\cdot$ Mitochondria $\cdot$ Bcl-2 family
Abbreviation
BOPs $\quad$ BH3 only proteins
pBcls proteins of the Bcl-2 family
MOM mitochondrial outer membrane
ART Apoptosis Regulating Target domain
CT C-terminal end
NT N-terminal end
CLIC Cytosolic Locked In Conformation
CLAC Cytochrome $c$ Liberation Associated Conformation 


\section{Introduction}

The mitochondrial integration of the many signals arising from different cellular compartments and functional pathways during apoptosis are essential for the completion of this cell death programme. This integration as well as the execution of apoptosis is controlled by the $\mathrm{Bcl}-2$ proteins, (pBCls), some of which undergo a change of conformation and cellular localization at the onset of apoptosis [1]. Two proapoptotic proteins of this family, Bax and Bak, are essential for the completion of apoptosis. In the case of Bax, the induction of apoptosis and the associated change in its cellular localization reflects the ability to undergo a conformational change from a soluble to an integral membrane conformation [2]. Numerous works have shown that this conformational change is dependent of both the expositions of the C-terminal and the N-terminal extremities of Bax, which are contemporary of Bax mitochondrial insertion into the outer membrane of mitochondria. Current models predict that the conformational changes involved in the translocation of Bax toward the organelle are associated with profound changes in secondary and, especially, tertiary structure of the protein. Given the importance of the biological activities of Bax the latter process is likely to be a major regulatory checkpoint for apoptosis and a putative major pharmacological target in numerous diseases [3].

\section{Existence of two major stable conformational states of Bax}

Bax is usually considered to adopt two stable conformational states: native Bax, present in the cytosol (therefore called CLIC for "Cytosolic Locked In Conformation") and fully activated Bax, related to the mitochondrial permeabilization (therefore called CLAC for "Cytochrome c Liberation Associated Conformation").

In aqueous solutions, Bax appears as a globular protein [4] with a structure very similar to that of some of the other pBcls, either proapoptotic (Bid and p15tBid) or antiapoptotic (Bcl-xl and Bcl-2) [5]. Bax is composed of 9 alpha helices separated by rather unstructured loops. Two central helices ( $\mathrm{H} \alpha 5$, and in a lesser extend $\mathrm{H} \alpha 6$ ) are mostly hydrophobic. They constitute the core of the protein and are embedded within the other 7 helices, which have amphipathic properties and keep their hydrophilic residues exposed to the exterior. This organization enables the protein to remain soluble in its native cytosolic conformation. Yet, some authors have reported the existence of mitochondria-associated Bax in healthy cells. This apparent discrepancy is discussed below.

Helices $\alpha 5$ and $\alpha 6$ are surrounded on one side by helices $\alpha 1$ and $\alpha 2$, on the other side by helices $\alpha 3$ and $\alpha 4$ and above

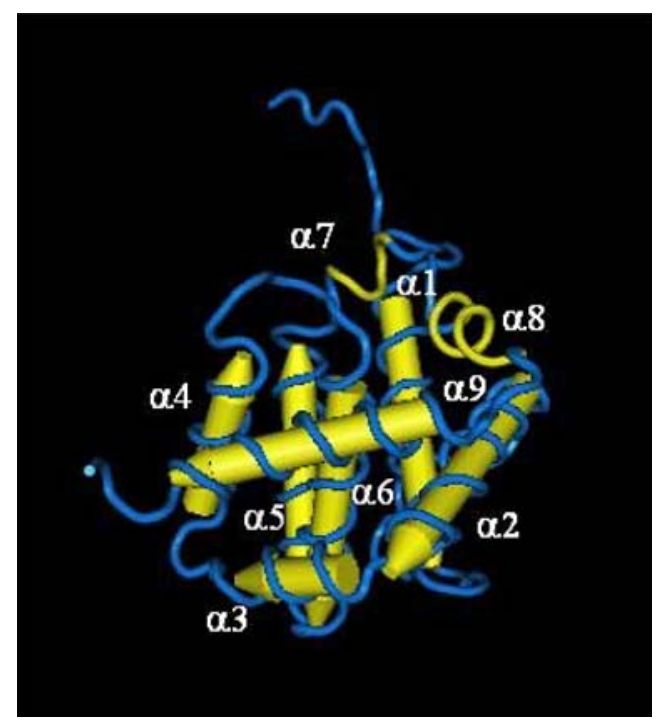

Fig. 1 The CLIC Bax closed-packed conformation, as described by Suzuki et al. [4]. The $9 \alpha$ helices are represented in yellow. The mostly hydrophobic $\alpha 5$ and $\alpha 6$ helices are embedded in the core of the protein. The C-terminal $\mathrm{H} \alpha 9$ is sequestered in the hydrophobic cleft, defined by the BH1, 2 and 3 domains, respectively overlapping the $\mathrm{H} \alpha 4-\mathrm{H} \alpha 5$ junction, the $\alpha 2$ helix and the $\alpha 7-\alpha 8$ helices

by $\mathrm{H} \alpha 7$ and $\alpha 8$ (Fig. 1). This structure is very similar to that determined for anti-apoptotic proteins pBcls, namely Bcl-2 and Bcl-xl, by crystallography or NMR studies [5]. NMR studies revealed an ordered conformation of $\mathrm{H} \alpha 1$ and $\mathrm{H} \alpha 2$ along the $\mathrm{H} \alpha 5-\alpha 6$ backbone. Helices are linked by a long, poorly structured loop (18 residues). Similarly to Bcl-xl, a fragment of Bax H $\alpha 1-\alpha 2$ loop (Glu44-Ala46) seems to establish bonds with residues of H $\alpha 6$ (Ile133 and Met137), suggesting that the loop participates to the compacted structure observed in solution [4] (Fig. 1).

On the surface of both Bcl-2 and Bcl-xl, the three highly conserved homology domains (BH1, 2 and 3) define the entry of an elongated hydrophobic pocket. This structural occurrence has been largely assigned as the basis of their antiapoptotic function since this hydrophobic cleft was shown to mediate the interaction with other $\mathrm{BH} 3$-proteins. The 3 Bcl-2-homology domains present in Bax sequence (BH1, 2 and 3) also define a hydrophobic pocket although Bax $\mathrm{BH} 3$ domain is packed closer to the core of the protein than the homologous domain of the anti-apoptotic pBcls [4]. Bax hydrophobic pocket is occupied in its native state by the hydrophobic helix $\alpha 9$ of the protein and several of its polar residues (i.e. Thr172, Thr174, Thr182, Thr186) are exposed to solvent, increasing the protein solubility. By analogy to the C-terminal transmembrane domain of Bcl-2 and $\mathrm{Bcl}-\mathrm{xl}$, this helix was proposed to be a sequence responsible for Bax anchoring to the MOM, but it is clear that in the native conformation resolved by Suzuki et al. [4] this domain is tightly sequestered. 
At last, a short sequence is highly conserved in the BH1 domain, namely the residues NWGR at the N-terminal end of $\mathrm{H} \alpha 5$. Especially the $\mathrm{W}$ residue (Trp) was shown to establish hydrophobic links with residues on $\mathrm{H} \alpha 7-8$, (i.e. $\mathrm{BH} 2$ domain) [5]. Giving the high sequence homology between Bax $\mathrm{H} \alpha$ 7-8 and Bcl-xl H $\alpha$ 7-8, it seems highly probable that similar links participate to stabilize Bax water-soluble structure.

Thus, the described conformation of native, cytosolic Bax suggests a closely-packed, globular protein. It seems unlikely that hidden domains of the protein can interact with other proteins unless undergoing some conformational modifications, relaxing the structure and enabling novel exogenous or endogenous interactions.

During apoptosis, major conformational change occurs, making Bax switch from a cytosolic to a mitochondrial, membrane-inserted protein. In this activated form, several domains, formerly hidden in the native conformation, become exposed. In particular, hydrophobic domains are accessible, increasing Bax affinity for biological membranes. The exposure of these domains upon the induction of apoptosis, which has been monitored by epitope-specific antibodies mainly by Richard Youle's group, provides structural insight to the activation of $\mathrm{Bax}$ [6-9]. The induction of conformational change of Bax by $\mathrm{pH}$ or detergent provokes a similar pattern of epitopes' exposure to that observed during apoptosis, suggesting that the activated Bax represents an alternative stable conformation [7, 10]. First, in all cases, the exposure of the N-terminal part of $\mathrm{Bax} \mathrm{H} \alpha 1$ (residues 12-24, recognized by the widely used 6A7 antibody) came together with Bax ability to homo- or hetero-dimerize with Bcl-xl. Further studies indicated that other domains are also exposed in detergent-activated Bax, namely the $\mathrm{BH} 3$ domain and the $\mathrm{N}$-terminal part of the $\mathrm{H} \alpha 5$ as assessed by the use of epitopespecific antibodies $[11,12]$. This suggests that the globular form of the protein is totally abolished when the protein is undergoing a type of "inside-out" transition before (or during) membrane integration. It should be noted however, that differences might exist when the structures in micelles and in phospholipids bilayer are compared to each other and that the membrane-associated conformation of Bax has not been structurally resolved yet.

However, among the domains of the protein exposed in the "activated" protein, several have been suggested to play an important role in the protein function.

The N-terminal part of Bax (i.e. Aa20-37) was shown to contain a mitochondrial targeting sequence: the first $\alpha$ helix sequence is able to target RFP to the mitochondria and inversely, the deletion of $\mathrm{Bax} \mathrm{H} \alpha 1$ abolishes its mitochondrial localization [13]. Yet, this sequence is insufficient to trigger the protein insertion into the mitochondrial outer membrane [13]. Of note, the first 20 amino-acids of Bax sequence play an inhibitory role for Bax mitochondrial localization and as been called "Apoptosis Regulating
Target" domain (ART) [14]. Interestingly, the targeting can be instated by deleting the domain or by replacing the Bax C-terminal helix with that of Bcl-2 [14], suggesting a close cooperation between both $\mathrm{N}$ - and C-terminal extremities of Bax. Of note, the junction between the ART and the addressing domain encompasses the epitope recognized by the anti-conformational antibody $6 \mathrm{~A} 7$.

Bax BH3 domain is believed to be another important domain in the protein, partly based on the observation that mutations or deletions of $\mathrm{BH} 3$ residues impair Bax binding properties and/or pro-apoptotic function [15]. Moreover, the $\mathrm{BH} 3$ domain is present in all pro-apoptotic pBcls, and in most of their anti-apoptotic counterparts. It was first thought to be involved in the ability of these proteins to form homoor hetero-oligomers. This assumption was demonstrated in the case of Bcl-2 and Bcl-xl binding of other BH3 proteins $[16,17]$. Indeed, the $\mathrm{BH} 3$ peptides which mimic $\mathrm{Bax} \mathrm{BH} 3$ domain are able to interact with the hydrophobic groove present on the surface of these proteins and formed by the $\mathrm{BH} 1,2$ and 3 domains. Besides, Bax BH3 peptide is able to inhibit Bax-Bcl-xl interaction, what insists on the specificity of the BH3-dependent binding [18].

The disruption of the Bax globular, closely-packed structure also suggests exposure of the $\mathrm{H} \alpha 5-\mathrm{H} \alpha 6$ hairpin, which is otherwise profoundly embedded in the native conformation. These helices are similar to the pore-forming domain of colicins and diphteria toxin. Mutagenesis experiments conducted by Nouraini et al. [19] showed that the central alpha helices, $\mathrm{H} \alpha 5-\alpha 6$, are the potential pore-forming domains of Bax. More recent studies showed that $\mathrm{H} \alpha 5-\alpha 6$ are transmembrane domains $[20,21]$ and that they are necessary to Bax insertion into the MOM, but not for its addressing to the organelle [11]. Both isolated $\alpha 5$ and $\alpha 6$ helices of Bax (and from other $\mathrm{pBcls}$ ) were shown to insert into synthetic lipid membrane where they form pores [21, 22].

The last potentially important domain exposed in detergent-treated Bax is the $\mathrm{C}$-terminal $\alpha 9$ helix. This helix is bound to Bax hydrophobic groove in the native conformation and is thought to participate to the conformational stability. Of note, only one polar residue of $\mathrm{H} \alpha 9$ faces towards the hydrophobic groove, namely S184, while the others are susceptible to increase Bax solubility [4]. Mutation, deletion or phosphorylation of S184 influences Bax localisation [23, 24]. By homology to anti-apoptotic proteins Bcl-2 and Bcl-xl, the $\alpha 9$ helix was proposed to be the tail-anchoring sequence for Bax mitochondrial localisation. This role is still currently discussed, since Bax mitochondrial translocation can occur despite of C-terminal deletion in some systems [13, 25]. Nevertheless, Bax $\mathrm{H} \alpha 9$ was shown to be involved in $\mathrm{Bax}$ insertion into pure lipid membranes and in the subsequent pore formation.

Both CLIC and CLAC are very stable conformational states of the protein but, it is not clear that they are the 
only physiological Bax conformers. Indeed, many studies revealed that intermediate conformations could be observed, some of them being reversible., Hsu et al. [7] pointed out on the fact that distinct detergents could produce various effects on Bax conformation and its ability to form dimers. The authors actually described 3 different stages in Bax conformation, as judged by its ability to form dimers and to expose the 6A7 epitope. Stage 1, observed without detergent or with zwitterionic detergent (CHAPS), corresponds to the native (CLIC) conformation, Bax does not interact with the 6A7antibody and is unable to dimerise. Stage 2 is observed when Bax is treated with anionic detergents or non ionic detergents with alkyl (W1) or acyl sorbitan ester groups (TWEEN 20); it is 6A7-positive and is capable to hetero-dimerise, but not to form homo-dimers. Finally, stage 3 is the activatedlike conformation where Bax is 6A7-positive and can homoor hetero-dimerise (CLAC). The latter stage is obtained by treating Bax with non ionic, alkylphenyl moieties-containing detergents. These compounds might force their entry into the Bax hydrophobic groove, inducing a sufficient perturbation of the structural stability and in this way trigger the conformational transition.

Some other groups reported slight conformational modifications of Bax which do not support cell death induction and are sometimes reversible $[10,12]$. These studies report physiologically relevant events, either a sub-lethal apoptotic stimulus or a change in the protein environment $[10,12]$, namely a subtle $\mathrm{pH}$ modification, previously described to occur during the induction of apoptosis [26]. Both studies suggest that these conditions can result in the exposure of some epitopes without the release of mitochondrial cytochrome $c$ and cell death. In both cases, the N-terminal $\alpha 1$ helix becomes accessible, followed by Bax translocation to the mitochondria. Makin et al. [12] have reported the formation of a mitochondrial complex including Bax, with an apparent size of $46 \mathrm{kD}$, thus not excluding homodimerisation. Cartron et al. [10] also showed that exposure of the C-terminal part of $\mathrm{H} \alpha 1$ and that of BaxBH3 occur simultaneously. Secondary, the Nterminal part of $\mathrm{H} \alpha 1$ and $\mathrm{H} \alpha 5$ become exposed, concomitant with Bax translocation and insertion into the MOM, inducing cytochrome $c$ release. This step is accompanied by a conformational transition during which the protein becomes resistant to mild proteolysis (Fig. 2).

We propose that Bax can adopt two stable conformational states, either inactive and soluble (CLIC), or activated, integrated into the MOM and responsible for cell death (CLAC). Nevertheless, in the cell, Bax is submitted to many environmental factors, varying in time, which slightly loosen the globular structure and enable protein-protein interactions. This loosening of the structure might be a required, early event necessary but not sufficient to induce Bax conformational transition under physiological conditions. The further binding of membrane compounds or of other BH3-
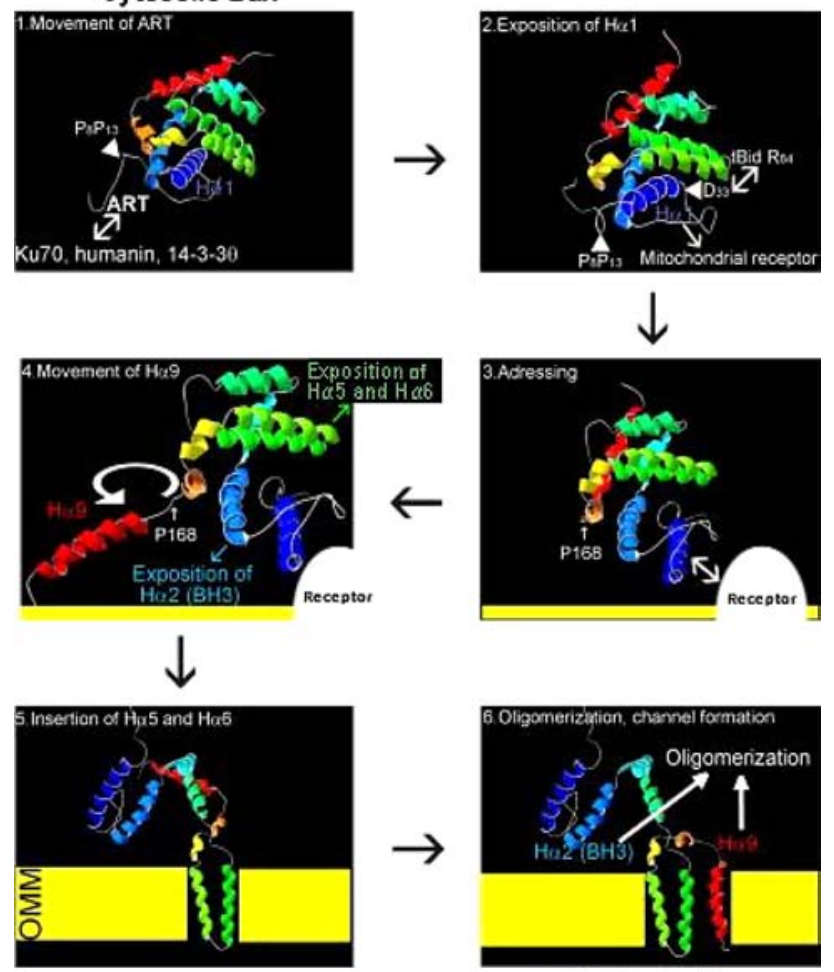

Membrane Bax
Cytosolic Bax

Fig. 2 Representation of the Bax CLIC/CLAC transition. (1) In Bax CLIC, the addressing sequence is hidden by the $\mathrm{N}$ - and the C-ART sequences; the N-ART domain can interact with inhibitory proteins and its position is controlled by the residues $\mathrm{P} 8$ and $\mathrm{P} 13$ which confer a rigid structure to the region. (2) tBid can disrupt the intramolecular bond between BaxD33 and BaxK64, thereby inducing the exposure of the mitochondrial addressing sequence. (3) Bax interacts with a protein receptor located at the outer mitochondrial membrane (OMM). (4) A rotation of the $\mathrm{H} \alpha 9$ around the $\mathrm{P} 168$ residue results in its liberation from the hydrophobic groove. This torsion of the protein induces the exposition of the $\alpha 5-\alpha 6$ helices and of the BH3 domain. (5) The hydrophobic $\alpha 5-\alpha 6$ helices insert into the OMM. The interaction with the mitochondrial receptor is disrupted. Bax association to the mitochondria becomes resistant to an alkaline treatment. (6) Bax CLAC homo-oligomerisation happens simultaneously to channel formation. The $\alpha 9$ helix insertion into the OMM participates to Bax oligomerisation and channel formation. Oligomerisation also implicates $\mathrm{Bax} \mathrm{BH} 3$ domain, possibly through a $\mathrm{H} \alpha 1-\mathrm{BH} 3$ interaction

containing proteins might be the determining event triggering the conformational change.

\section{Bax interaction with membranes}

\subsection{Interaction with membranes}

Bcl-2 and $\mathrm{Bcl}-\mathrm{xl}$ both present a hydrophobic C-terminal tail which has been shown to be necessary to their association to membranes. Similarly, the Bax C-terminal end is a quite hydrophobic $\alpha$ helix. It was proposed that $\mathrm{Bax} \mathrm{H} \alpha 9$ could be responsible for Bax mitochondrial targeting during 
apoptosis. Wolter et al. [6] showed that GFP-Bax translocation during apoptosis was abrogated when the 21 last aminoacids were deleted and that lack of binding was not due to an increased interaction of full-length GFP-Bax with Bcl-2 or Bcl-xl. Nechustan et al. [23] later established that a serine in position 184 determined Bax localisation. Indeed, the mutation of S184 to a charged amino-acid (K, D or E) abolished Bax mitochondrial targeting even under apoptotic conditions. Interestingly, it was later shown that this inhibition, due to a charged residue, was reproduced by AKT-induced phosphorylation of S184 [24]. Conversely, S184 deletion or mutation to valine induced a constitutive mitochondrial localisation, unrelated to apoptosis induction [23].

Bax insertion into ER membrane vesicles [27] and pure lipid micelles [25] was also tested in vitro. These experiments show that 3 sequences in Bax were potential membraneanchors, namely the $\mathrm{H} \alpha 5-\alpha 6$ hairpin, $\mathrm{H} \alpha 1$ and $\mathrm{H} \alpha 9$. Under non apoptotic conditions, Bax monomer seems to associate at the same level to either ER (endoplasmic reticulum) liposomes or mitochondrial liposomes [28] suggesting that Bax association with membrane lipids is not absolutely dependent on the presence of cardiolipin in healthy cells.

The interaction between $\mathrm{H} \alpha 9$ and membrane lipids thus seems to be responsible for the membrane localisation of Bax [28], despite being not specific for mitochondria since Bax can also be found on ER in these systems. Moreover, Bax binding to the membranes through its C-terminal $\alpha$ helix is not sufficient to induce apoptosis, even though it can sometimes appear necessary [20]. To induce apoptosis, Bax has to undergo an additional conformational change responsible for a more complete insertion, as reported in cells [29] or in acellular systems [20,30]. This conformational transition comes together with Bax oligomerisation. The previous observations are actually consistent with the hypothesis that $\mathrm{H} \alpha 9$ removal from the Bax hydrophobic groove could facilitate Bax activation, if not even trigger it. Confirming this assumption, we recently reported that point mutations on Bax $\mathrm{H} \alpha 9$ (P168V and S184V), previously shown to remove $\mathrm{H} \alpha 9$ from the groove formed by the central helices [23], induced a slight modification in Bax conformation, but yet not enough to activate the protein [11] (Fig. 2).

\subsection{Specific interactions with mitochondria}

The mitochondrial outer membrane is definitively not a simple lipid membrane. First, the MOM lipid composition is not quite specific. Second, many membrane-associated and membrane-inserted proteins are part of the MOM [31].

In healthy HeLa cells, Bax is constitutively partially mitochondrial. Bax activation is therefore monitored by its membrane-insertion and oligomerisation [29]. In this cellular system, Roucou et al. [32] showed that Bax oligomerisation induced by $\mathrm{tBid}$ was dependent on a mitochondrial protein which is different from VDAC. Consistent with this, Kuwana et al. [28] observed in a stepwise simplified system of reconstituted vesicles that Bax mitochondrial oligomerisation induced by tBid happened in MOM vesicles and not in mitochondrial liposomes. This work suggests that $\mathrm{tBid}$-induced Bax oligomerisation requires a MOM protein partner, drastically improving Bax-dependent vesicle permeabilisation. This assumption has been otherwise demonstrated, as described below.

Contrary to other proteins from the family, Bax contains a mitochondrial targeting sequence included in the $\mathrm{N}$ terminal $\mathrm{H} \alpha 1$ (Aa20-37) [13]. A Bax variant lacking the 19 first residues, thus similar to Bax $\triangle \mathrm{ART}$ [14], called Bax $\Psi$ is constitutively bound to the mitochondria in some nervous system tumors [33]. Our group demonstrated that a chimera protein, built from this 18 amino-acids sequence addition to the N-terminal end of a cytosolic protein (RFP), became mitochondria-associated [13]. Strikingly, Bax Nterminal targeting to the MOM was inhibited by the addition of Aa20-37-RFP in a dose-dependent manner, while RFPBax CT $(\Delta S 184)$ had no inhibitory effect, although exhibiting a mitochondrial localisation. This competition between the first two compounds suggests the existence of a rate-limiting receptor for the binding of Bax N-terminal addressing sequence. Yet, $\mathrm{H} \alpha 1$ mitochondrial addressing is not sufficient to induce Bax mitochondrial insertion and the subsequent mitochondrial permeabilisation. Indeed, mutations on $\mathrm{H} \alpha 5-\alpha 6$ abrogating their pore-forming activity prevented $\operatorname{Bax} \Delta 1-19$ from membrane insertion and toxicity [13].

Similarly, Makin et al. [12] reported a change in Bax Nterminal conformation which was reversible and not always accompanied by cytochrome $c$ release. This implies that the Bax conformational switch does not happen, since fully activated Bax (i.e TritonX100-treated or octylglucoside-treated Bax) provokes mitochondrial permeabilisation and cell death [30, 34]. This study therefore suggests that Bax homodimerisation can occur at the MOM without its conformational switch, or that another protein with a similar molecular weight might dimerise with Bax at the MOM in a reversible manner.

Bax N-terminal and C-terminal ends seem to be able to mediate its association to the mitochondria but the biological significance of both signals appears different. Indeed, according to Bax resolved structure [4], the exposure of its C-terminus correlates with the exposure of Bax hydrophobic groove, thus enabling further interactions with mitochondrial membrane. The Bax C-terminus could thus be regarded as an inhibitory domain for Bax interaction with other Bcl-2family proteins and consequently, as proposed by Arokium et al. [11], would constitute a second ART domain (C-ART). Interestingly, both $\mathrm{N}$ - and $\mathrm{C}$-ART seem to participate to the native conformation stabilisation since the double deletion $(\Delta 1-19, \Delta H 9)$ increases the toxicity of N-ART deleted Bax 
[11]. Thus, Bax targeting to mitochondria might involve simultaneous or sequential removal of both N-ART and CART inhibitions.

There must be a first event unmasking the addressing sequence located within the first alpha helix [13]. Actually, this stimulus is most often sufficient to trigger Bax activation. A possible explanation to this is that the $\mathrm{H} \alpha 1$ takes part to stability of the hydrophobic groove as removing N-ART and $\mathrm{H} \alpha 1$ away from the core of the protein may be sufficient to a profound destabilization of the protein structure leading in particular to the exposure of the Bax BH3 domain.

To conclude this chapter, Bax targeting to the mitochondria seems to be necessary but not sufficient to induce a conformational transition to a fully active Bax conformation but would rather decrease the activation threshold of the protein.

\section{Bax interaction with BH3-only proteins}

BH3-only proteins are a sub-group of the Bcl-2 family. They share the particularity to bear only one homology domain to Bcl-2 (BH3). This BH3 domain is exposed in all BH3-only proteins (BOPs) except Bid [35]. As a consequence, BOPs are either transcriptionally (Puma, Noxa) or post-translationally inhibited (Bad, Bim) in healthy cells. As an exception, Bid exists as an inactive, cytosolic protein in healthy cells and undergoes a proteolytic cleavage responsible for its activation (tBid) and $\mathrm{BH} 3$ exposure during apoptosis. BOPs are incapable of inducing cell death in the absence of both Bax and Bak. Their function in apoptosis can be resumed by their BH3 domain and mimicked by BH3-peptides [35], which suggests that $\mathrm{BH} 3$ is the critical domain for their interaction with other proteins from the family and for their function.

tBid was shown to directly induce Bax activation, whereas some other BOPs function (Bad, Noxa) is rather mediated by anti-apoptotic proteins (Bcl-2, Bcl-xl or Mcl-1). BOPs could thus be split in two categories: activators of multidomain proteins (Bid-like) and repressors of anti-apoptotic proteins (Bad-like) [35].

We will use the tBid-Bax interaction as a model: tBid was shown to provoke a conformational change at the Bax $\mathrm{N}$-terminal end, enabling it to form mitochondrial oligomers and to induce MOM permeabilization [36, 37]. This activation is dependent on Bid BH3 integrity and can be reproduced by the use of a sole peptide encompassing for Bid BH3 domain [37, 38]. The interaction between Bcl-2-like proteins and $\mathrm{BH} 3$ peptides or proteins is mediated by the hydrophobic groove. It is thus tempting to postulate that the interaction between $\mathrm{tBid}$ and Bax could involve the Bax hydrophobic cleft. Wang et al. [39] demonstrated that Bid interaction with either Bcl-2 or Bax was conditioned to Bid$\mathrm{BH} 3$ and Bcl-2- or Bax-BH1 integrity. Besides, the removal of Bax C-terminal $\mathrm{H} \alpha 9$ does not impair tBid-induced function, even if pore formation is altered [40]. Consistent with this, Yethon et al. [30] observed that incubating Bax with tBid induced no conformational change of Bax unless liposomes were added, possibly sequestering $\mathrm{Bax} \mathrm{H} \alpha 9$. In the same idea, Bax detergent-induced unfolding [40] or mutational activation (Bax $\triangle \mathrm{ART}$ and $\mathrm{Bax} \mathrm{P} 13 \mathrm{~V})$ [41] decreases tBid ability to interact with Bax and induce its membraneinsertion. The Bax hydrophobic groove is thus likely to take part to tBid-Bax interaction.

To further characterize Bax-tBid interaction, we used mutagenesis strategies and pull-down experiments between Histagged tBid and Bax to demonstrate the critical role of 3 residues of Bid BH3 in tBid-Bax interaction, namely R84, G94 and M97 [42]. We next observed that $\mathrm{Bax} \mathrm{H} \alpha 1$ alone was able to interact with BidBH3 and with tBid, whereas a point mutation D33A in this helix abolished the ability of BidBH3 and tBid to interact with $\mathrm{Bax}(\mathrm{D} 33 \mathrm{~A})$ or $\mathrm{Bax} \mathrm{H} \alpha 1$ (D33A). Of note, Puma and PumaBH3 were all the same capable of binding $\mathrm{H} \alpha 1$ in the presence of its D33 residue, while neither $\mathrm{Bad}$ nor BadBH3 could. The specificity of this interaction is underlined by the inhibitory effect of a peptide encompassing $\mathrm{BaxH} \alpha 1$ on tBid-Bax binding. Since both D33 (Bax H $\alpha$ ) and R84 (Bid BH3) residues play a critical role in the interaction, we wondered if the mutation of one of these charged residues could be conteracted by a mutation of the other one. As hypothesized, while BaxD33R and tBidR84D could not interact with wt-tBid and wt-Bax respectively, the interaction was restored between BaxD33R and tBidR84D. This shows that Bax interacts with $\mathrm{tBid}$ through an electrostatical interaction involving the D33 (Bax $\mathrm{H} \alpha 1)$ and the R84 (Bid BH3) residues [42] (Fig. 3). We later demonstrated that such an electrostatic interaction exists in the inactive conformation of Bax between the BaxD33 and BaxK64 residues, the latter being part of the Bax BH3 domain [11]. Artificial disruption of this bond through the mutation of one of these residues results in a slight conformational change of the protein, as observed by the exposure of normally hidden epitopes, namely the BH3 (residues 57-72) and the C-terminal part of $\mathrm{H} \alpha 1$ (residues 24-33) [11] (Fig. 3).

We propose a model in which tBid could interact with the Bax hydrophobic groove and with $\mathrm{Bax} \mathrm{H} \alpha 1$. The first hypothesis is that $\mathrm{BidBH} 3$ removes $\mathrm{BaxH} \alpha 9$ from the groove, thereby initiating the conformational change, with a subtle displacement of the BH1 domain (cf paragraph 2.1). Once in the groove, high physical proximity between BaxD33, BaxK64 and BidR84 could disrupt the stabilizing bond D33K64, thereby achieving the unfolding of the hydrophobic groove and the conformational transition of the protein. This implies that the $\mathrm{N}$-terminal extremity of BidBH3 is located next to BaxBH3, i.e. with a parallel orientation to BaxBH3. This disagrees with the binding described between Bcl-xl and BakBH3 [43]. The second hypothesis is that tBid could 

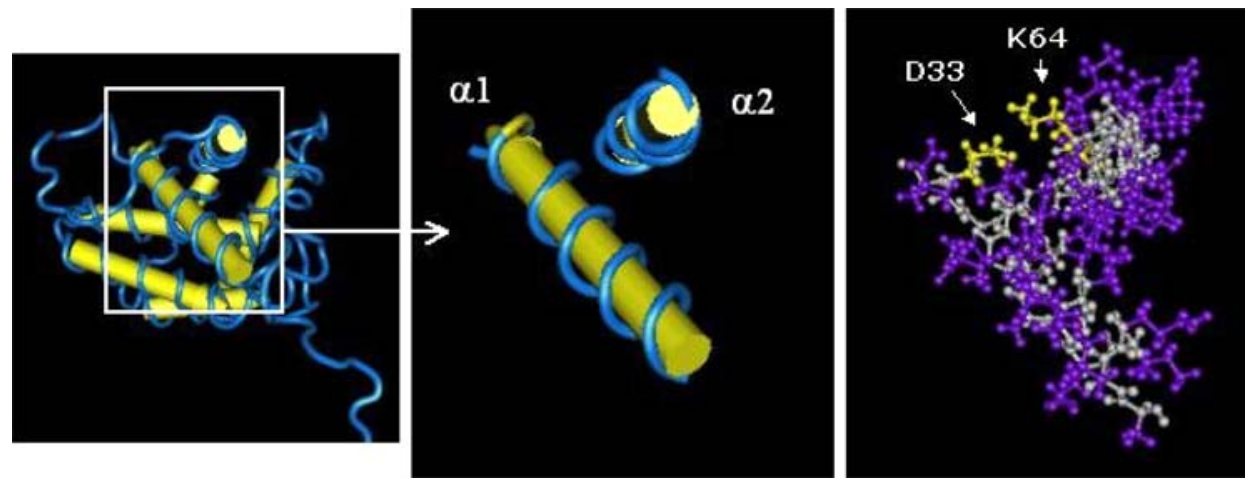

Fig. 3 Illustration of the electrostatic bond between the $\mathrm{Bax} \mathrm{H} \alpha 1$ and BH3 domain. The 2 first helices of Bax are isolated (left and middle panels). The right panel shows the postition of the D33 and K64 residues of Bax (gray: protein backbone; purple: accessory chains; yellow: D33

approach the solvent-exposed side of $\mathrm{BaxBH} 3$ to disrupt the Bax D33-K64 electrostatic bond, whereby the G94 and M97 residues of Bid stabilise the interaction with Bax. The disruption of the intramolecular bond could move $\mathrm{H} \alpha 1$ away from the rest of the protein. The displacement of $\mathrm{H} \alpha 1$ might be a sufficient event to trigger Bax unfolding, as suggested by the effects of calpain cleavage [42]. The disruption of the Bax D33-K64 bond might be sometimes completed by a concomitant loosening of Bax hydrophobic groove, finalising the destabilisation of the Bax conformation. This is consistent with the observation suggesting a cooperation between liposomes, potentially sequestering $\mathrm{H} \alpha 9$, and $\mathrm{tBid}$ in recombinant Bax activation [30].

Once Bax is activated, Bid affinity to the activated protein drastically decreases and Bid is released from the dimer, illustrating the so-called "hit-and-run" model of Bid-induced activation. This is in agreement with the fact that Bid is not found in Bax mitochondrial complexes during late apoptosis.

\section{Bax interaction with anti-apoptotic proteins from the Bcl-2 family}

\subsection{Bax binding to the proteins}

Bax interaction with anti-apoptotic proteins from the Bcl-2 family (Bcl-2, Bcl-xl, Mcl-1, A1) was already described in 1995 by Sedlak et al. [44]. Bcl-2-like anti-apoptotic proteins were later shown to function through sequestering the $\mathrm{BH} 3$ domain of BH3-containing proteins in their hydrophobic cleft, formed by BH1, 2 and 3 [45]. This interaction did not appear to be very specific for the $\mathrm{BH} 3$ domain, since substitution of Bax BH3 by Bcl-2 $\mathrm{BH} 3$ did not alter the binding [46]. Despite of this, several residues in BaxBH3 have been shown to play a role in BaxBH3 binding to $\mathrm{Bcl}-2$ or Bcl-xl, or deduced by sequence homology through the study of BakBH3 binding to the same proteins [43]. Namely, and K64). An electrostatic interaction between the negatively-charged D33 and the positively-charged K64 ensures the sequestration of the $\mathrm{H} \alpha 1$ near the $\mathrm{BH} 3$ domain

Bax residues D68, D71, E69 respectively establish hydrogen bonds with G145, G145 and R146, R107 on Bcl-2 and R139, G138, R100 on Bcl-xl. Among these residues, D68 and E69 appear to be particularly critical to Bcl-2 binding [46]. Besides, Bax hydrophobic residues K64, I66 and L70 are believed to face the hydrophobic groove of the anti-apoptotic proteins. Hirotani et al. also observed a role of Bcl-2 and Bcl-xl BH4 domain in their Bax binding ability [47]. Given the known structure of Bcl-2 and Bcl-xl, BH4 participates in stabilizing the hydrophobic groove. The deletion of $\mathrm{BH} 4$ or the mutation of some residues into charged amino-acids can stand for a change in the shape of the hydrophobic cleft, which may account for the loss-of-function of the protein.

It is noteworthy that the observation of Bax interaction with $\mathrm{Bcl}-2$ or $\mathrm{Bcl}-\mathrm{xl}$ under non apoptotic conditions sometimes requires the presence of detergents. Moreover, Bcl-2like proteins have a greater binding capacity to Bax $\Delta 1-19$ and BaxD33A, as previously described. This fits well with our previous hypothesis that Bax structure loosening makes protein-protein interaction more likely. This also suggests that Bcl-2-like proteins can act either upstream or downstream Bax activation to inhibit its oligomerisation and the subsequent mitochondrial permeabilization.

Inversely, modifications responsible for alterations of the hydrophobic cleft alter the interactions between Bax and Bcl-2-like proteins. For instance, point mutations of residues L130, G138, G159 (Bcl-xlBH1), Y195 (Bcl-xlBH2) or Y101 (Bcl-xlBH3) have all been demonstrated to impair the binding $[44,48]$.

It was recently shown that $\mathrm{Bcl}-2$ interaction with the proapoptotic proteins (Bid or Bax in the study) induced a change in its conformation, responsible for a more complete insertion in the MOM [49].

Some anti-apoptotic proteins, especially $\mathrm{Bcl}-\mathrm{xl}$, are capable of forming reversible homo-dimers in the cytosol of healthy cells. Jeong et al. [50] demonstrated that these dimers involved the binding of the C-terminal $\alpha$ helix of 
one protein to the hydrophobic groove of the second one. Since the Bax structure is very similar to that of Bcl-xl under native conditions, Jeong et al. [50] reported a possible hetero-dimerisation between $\mathrm{Bax}$ and $\mathrm{Bcl}-\mathrm{xl}$ involving the replacement of $\mathrm{Bax} \mathrm{H} \alpha 9$ by $\mathrm{Bcl}-\mathrm{xl} \mathrm{CT}$ in the hydrophobic cleft. Yet, this interaction was only seen in the presence of detergents or with a C-truncated form of Bax, suggesting that a first modification in the structure of Bax is necessary to unlock the protein.

This type of interaction may be an alternative to the widely described binding of BaxBH3 domain in the hydrophobic cleft of anti-apoptotic proteins.

\subsection{Disruption of the interaction}

While studying the mechanism by which BOPs participate to Bax (and Bak) activation, some groups discovered that the interaction between Bax and Bcl-2-like proteins could be disrupted by natural (BOPs) or artificial compounds (BH3 peptides or non peptidic compounds) [35, 38, 52-55]. Quite strikingly, the disruption of this interaction can release activated Bax. The ability of $\mathrm{BH} 3$ peptides to release activated Bax from Bcl-xl was extensively studied by Moreau et al. [18]. This study demonstrates that native Bax incubated with $\mathrm{Bcl}-\mathrm{xl}$ could be activated by the disruption of this interaction induced by $\mathrm{BaxBH} 3$ or BadBH3. In this experimental setting, $\mathrm{Bcl}-\mathrm{xl}$ (or Bcl-2) can be seen as the necessary intermediates between some apoptosis inducers and Bax activation.

As previously proposed, we suggest that Bax interaction with anti-apoptotic Bcl-2-like proteins may require a first loosening of Bax structure, perhaps due to a physicochemical change of the protein cellular environment or to organic co-factors. This loosening of the structure is not sufficient by itself to induce Bax drastic conformational change, yet sufficient to enable protein-protein interactions. In particular, the removal of $\mathrm{BaxH} \alpha 9$ may allow $\mathrm{BaxBH} 3$ binding into $\mathrm{Bcl}-2$ hydrophobic pocket. The stable engagement of $\mathrm{BaxBH} 3$ inside the groove would confirm Bax remodelling and enable the release of activated Bax after Bax displacement from the groove by an antagonist of the interaction. The study by Duglosz et al. [49] provides an interesting insight to this hypothesis as they demonstrate that Bcl-2 inhibitory effect on Bax-induced mitochondrial permeabilization was dependent on a change in Bcl-2 conformation and insertion to the mitochondrial membrane through the $\mathrm{H} \alpha 5-\alpha 6$. Of note, a conserved sequence in the BH1 domain, at the N-terminal part of $\mathrm{H} \alpha 5$ (NWGR), was shown to determine the binding between Bcl-2 or Bcl-xl and BH3 domains [5]. The arginine residue (Bcl-xl R139 and Bcl-2 R146) is involved in the $\mathrm{BH} 3$ pro-apoptotic proteins binding while the tryptophan residue (Bcl-xl W137 and Bcl-2 W144) is in tight contact with hydrophobic residues of $\mathrm{BH} 2$ domain, among which Bcl-2 W188 appears crucial to the groove stability. It is con- ceivable that such a change in Bcl-2 conformation would induce a comparable change in the ligand. Bcl-2 $\mathrm{H} \alpha 5-\alpha 6$ engagement into the MOM should pull on BaxBH3 through Bcl-2R146/BaxD71 bond and induce the unfolding of Bax hydrophobic core. Bax H $\alpha$ 5- $\alpha 6$ would therefore be exposed and insert into the MOM, in parallel with $\mathrm{Bcl}-2 \mathrm{H} \alpha 5-\alpha 6$. $\mathrm{Bcl}-2$ would thus prevent Bax oligomerisation and the subsequent mitochondrial protein release.

This mechanism makes of Bcl-2-like proteins the mediators of some BOPs apoptotic function, helping them to induce cell death. This dual role of Bcl-2-like proteins might give rise to a new class of anti-cancerous therapeutic means, using the defensive arsenal of cancerous cells to selectively kill them.

\section{Conclusion}

The aim of this review was to propose some molecular explanations to the surprising discrepancy between the tightly locked, native conformation of Bax described and its propensity to interact with the other proteins from the Bcl-2 family. We suggest that the conformation described by Suzuki et al. [4] might be a frozen model of Bax structural organization, yet it might be less locked in the cell. In the cytosol, Bax is submitted to a varying environment in terms of $\mathrm{pH}$, ionic concentrations, ATP, NADH, oxygen and many other parameters. Environmental changes might induce stretches in Bax structure, loosening it and enabling the access of proteins to some critical regions of Bax. The interaction can either reduce the activation threshold of Bax and/or stabilize certain conformational states of the protein. Some anti-apoptotic proteins prevent Bax mitochondrial oligomerisation through sequestering Bax in the cytosol (Humanin, Ku70, 14-3-3 $\theta$ ), away from its target. Other proteins bind the active conformation of Bax and are found at the mitochondria, where they prevent Bax oligomerisation and the subsequent cytochrome $c$ release (clusterin, Bcl-2). Bax interaction with proteins can also result in its activation [2]. Some interactions namely induce a sufficient disorder to trigger Bax conformational switch. Several crucial domains of Bax become exposed, enabling its homo-oligomerisation. The modality of oligomerisation is still unknown, but some elements could be part of the explanation. First, we demonstrated that Bax $\mathrm{H} \alpha 1$ could bind Bax BH3, which could also create intermolecular bonds. Then, data suggest that Bax C-terminal $\mathrm{H} \alpha 9$ could participate to the pore formation [40], maybe through its polar residues. Finally, a study reported the possible formation of intermolecular disulfide bonds involving Bax C62 and C126 residues [56]. Bax mitochondrial localisation may create a favourable environment to the formation of such bonds. Any of these interactions, yet not exclusive, can stand for Bax oligomerisation but have to date not been demonstrated in pathophysiological conditions. 
Acknowledgments Authors of this review are supported by the ANR grant "molecular mechanisms of Bax activation," INSERM (FMV), CNRS (BB, SM) and the "Ligue National contre le Cancer" (FMV)

\section{References}

1. Adams JM, Cory S (2001) Life-or-death decisions by the Bcl-2 protein family. Trends Biochem Sci 26:61-66

2. Er E, Oliver L, Cartron P-F, Juin P, Manon S, Vallette FM (2006) Mitochondria as the target of the pro-apoptotic protein Bax. Biochimica et Biophysica Acta (BBA)-Bioenergetics 1757:1301-1311

3. Cartron PF, Juin P, Oliver L, Meflah K, Vallette FM (2003) Impact of proapoptotic proteins Bax and Bak in tumor progression and response to treatment. Expert Rev Anticancer Ther 3:563-570

4. Suzuki M, Youle RJ, Tjandra N (2000) Structure of Bax: coregulation of dimer formation and intracellular localization. Cell 103:645-654

5. Petros AM, Olejniczak ET, Fesik SW (2004) Structural biology of the Bcl-2 family of proteins. Biochim Biophys Acta 1644:83-94

6. Wolter KG, Hsu Y-T, Smith CL, Nechushtan A, Xi X-G, Youle RJ (1997) Movement of Bax from the Cytosol to Mitochondria during Apoptosis. J Cell Biol 139:1281-1292

7. Hsu YT, Youle RJ (1998) Bax in murine thymus is a soluble monomeric protein that displays differential detergent-induced conformations. J Biol Chem 273:10777-10783

8. Hsu YT, Youle RJ (1997) Nonionic detergents induce dimerization among members of the Bcl-2 family. J Biol Chem 272:13829-13834

9. Hsu YT, Wolter KG, Youle RJ (1997) Cytosol-to-membrane redistribution of $\mathrm{Bax}$ and $\mathrm{Bcl}-\mathrm{X}(\mathrm{L})$ during apoptosis. Proc Natl Acad Sci USA 94:3668-3672

10. Cartron PF, Oliver L, Mayat E, Meflah K, Vallette FM (2004) Impact of $\mathrm{pH}$ on $\mathrm{Bax}$ a conformation, oligomerization and mitochondrial integration. FEBS Lett 578:41-46

11. Cartron PF, Arokium H, Oliver L, Meflah K, Manon S, Vallette FM (2005) Distinct domains control the addressing and the insertion of Bax into mitochondria. J Biol Chem 280:10587-10598

12. Makin GW, Corfe BM, Griffiths GJ, Thistlethwaite A, Hickman JA, Dive C (2001) Damage-induced Bax N-terminal change, translocation to mitochondria and formation of Bax dimers/complexes occur regardless of cell fate. Embo J 20:6306-6315

13. Cartron P-F, Priault M, Oliver L, Meflah K, Manon S, Vallette FM (2003) The N-terminal end of Bax contains a mitochondrialtargeting signal. J Biol Chem 278:11633-11641

14. Goping IS, Gross A, Lavoie JN, Nguyen M, Jemmerson R, Roth K, Korsmeyer SJ, Shore GC (1998) Regulated targeting of BAX to mitochondria. J Cell Biol 143:207-215

15. Wang K, Gross A, Waksman G, Korsmeyer SJ (1998) Mutagenesis of the $\mathrm{BH} 3$ domain of $\mathrm{BAX}$ identifies residues critical for simerization and killing. Mol Cell Biol 18:6083-6089

16. Otter I, Conus S, Ravn U, Rager M, Olivier R, Monney L, Fabbro D, Borner C (1998) The binding properties and biological activities of Bcl-2 and Bax in cells exposed to apoptotic stimuli. J Biol Chem 273:6110-6120

17. Conus S, Kaufmann T, Fellay I, Otter I, Rosse T, Borner C (2000) $\mathrm{Bcl}-2$ is a monomeric protein: prevention of homodimerization by structural constraints. Embo J 19:1534-1544

18. Moreau C, Cartron PF, Hunt A, Meflah K, Green DR, Evan G, Vallette FM, Juin P (2003) Minimal BH3 peptides promote cell death by antagonizing anti-apoptotic proteins. J Biol Chem 278:19426-19435

19. Nouraini S, Six E, Matsuyama S, Krajewski S, Reed JC (2000) The putative pore-forming domain of Bax regulates mitochon- drial localization and interaction with $\mathrm{Bcl}-\mathrm{X}(\mathrm{L})$. Mol Cell Biol 20:1604-1615

20. Annis MG, Soucie EL, Dlugosz PJ, Cruz-Aguado JA, Penn LZ, Leber B, Andrews DW (2005) Bax forms multispanning monomers that oligomerize to permeabilize membranes during apoptosis. Embo J 24:2096-2103

21. Garcia-Saez AJ, Coraiola M, Serra MD, Mingarro I, Muller P, Salgado J (2006) Peptides corresponding to helices 5 and 6 of Bax can independently form large lipid pores. FEBS J 273:971981

22. Schendel SL, Montal M, Reed JC (1998) Bcl-2 family proteins as ion-channels. Cell Death Differ 5:372-380

23. Nechushtan A, Smith CL, Hsu YT, Youle RJ (1999) Conformation of the Bax C-terminus regulates subcellular location and cell death. Embo J 18:2330-2341

24. Gardai SJ, Hildeman DA, Frankel SK, Whitlock BB, Frasch SC, Borregaard N, Marrack P, Bratton DL, Henson PM (2004) Phosphorylation of Bax Ser184 by Akt regulates its activity and apoptosis in neutrophils. J Biol Chem 279:21085-21095

25. Antonsson B, Montessuit S, Lauper S, Eskes R, Martinou JC (2000) Bax oligomerization is required for channel-forming activity in liposomes and to trigger cytochrome $c$ release from mitochondria. Biochem J 345 (Pt 2):271-278

26. Khaled AR, Kim K, Hofmeister R, Muegge K, Durum SK (1999) Withdrawal of IL-7 induces Bax translocation from cytosol to mitochondria through a rise in intracellular $\mathrm{pH}$. Proc Natl Acad Sci USA 96:14476-14481

27. Garcia-Saez AJ, Mingarro I, Perez-Paya E, Salgado J (2004) Membrane-insertion fragments of Bcl-xL, Bax, and Bid. Biochemistry 43:10930-10943

28. Kuwana T, Mackey MR, Perkins G, Ellisman MH, Latterich M, Schneiter R, Green DR, Newmeyer DD (2002) Bid, Bax, and lipids cooperate to form supramolecular openings in the outer mitochondrial membrane. Cell 111:331-342

29. Antonsson B, Montessuit S, Sanchez B, Martinou JC (2001) Bax is present as a high molecular weight oligomer/complex in the mitochondrial membrane of apoptotic cells. J Biol Chem 276:11615-11623

30. Yethon JA, Epand RF, Leber B, Epand RM, Andrews DW (2003) Interaction with a membrane surface triggers a reversible conformational change in Bax normally associated with induction of apoptosis. J Biol Chem 278:48935-48941

31. Esposti MD, Dive C (2003) Mitochondrial membrane permeabilisation by Bax/Bak. Biochem Biophys Res Commun 304:455-461

32. Roucou X, Montessuit S, Antonsson B, Martinou JC (2002) Bax oligomerization in mitochondrial membranes requires tBid (caspase-8-cleaved Bid) and a mitochondrial protein. Biochem J 368:915-921

33. Cartron PF, Oliver L, Martin S, Moreau C, LeCabellec MT, Jezequel P, Meflah K, Vallette FM (2002) The expression of a new variant of the pro-apoptotic molecule Bax, Baxpsi, is correlated with an increased survival of glioblastoma multiforme patients. Hum Mol Genet 11:675-687

34. Cartron PF, Gallenne T, Bougras G, Gautier F, Manero F, Vusio P, Meflah K, Vallette FM, Juin P (2004) The first alpha helix of Bax plays a necessary role in its ligand-induced activation by the BH3-only proteins Bid and PUMA. Mol Cell 16:807-818

35. Juin P, Cartron PF, Vallette FM (2005) Activation of Bax by BH3 domains during apoptosis. Cell Cycle 4:637-642

36. Desagher S, Osen-Sand A, Nichols A, Eskes R, Montessuit S, Lauper S, Maundrell K, Antonsson B, Martinou JC (1999) Bid-induced conformational change of Bax is responsible for mitochondrial cytochrome $c$ release during apoptosis. J Cell Biol 144:891-901

37. Eskes R, Desagher S, Antonsson B, Martinou JC (2000) Bid induces the oligomerization and insertion of Bax into the outer mitochondrial membrane. Mol Cell Biol 20:929-935 
38. Letai A, Bassik MC, Walensky LD, Sorcinelli MD, Weiler S, Korsmeyer SJ (2002) Distinct BH3 domains either sensitize or activate mitochondrial apoptosis, serving as prototype cancer therapeutics. Cancer Cell 2:183-192

39. Wang K, Yin XM, Chao DT, Milliman CL, Korsmeyer SJ (1996) BID: A novel BH3 domain-only death agonist. Genes Dev 10:2859-2869

40. Terrones O, Antonsson B, Yamaguchi H, Wang HG, Liu J, Lee RM, Herrmann A, Basanez G (2004) Lipidic pore formation by the concerted action of proapoptotic BAX and tBID. J Biol Chem 279:30081-30091

41. Cartron P-F, Moreau C, Oliver L, Mayat E, Meflah K, Vallette FM (2002) Involvement of the N-terminus of Bax in its intracellular localization and function. FEBS Lett 512:95-100

42. Cartron PF, Gallenne T, Bougras G, Gautier F, Manero F, Vusio P, Khaled M, Vallette FM, Juin P (2004) The first helix of Bax plays a necessary role in its ligand-induced activation by the BH3-only proteins BID and PUMA. Mol Cell 16:807-818

43. Sattler M, Liang H, Nettesheim D, Meadows RP, Harlan JE, Eberstadt M, Yoon HS, Shuker SB, Chang BS, Minn AJ, Thompson CB, Fesik SW (1997) Structure of Bcl-xL-Bak peptide complex: recognition between regulators of apoptosis. Science 275:983-986

44. Sedlak TW, Oltvai ZN, Yang E, Wang K, Boise LH, Thompson CB, Korsmeyer SJ (1995) Multiple Bcl-2 family members demonstrate selective dimerizations with Bax. Proc Natl Acad Sci USA 92:7834-7838

45. Zha H, Aime-Sempe C, Sato T, Reed JC (1996) Proapoptotic protein Bax heterodimerizes with $\mathrm{Bcl}-2$ and homodimerizes with Bax via a novel domain (BH3) distinct from $\mathrm{BH} 1$ and $\mathrm{BH} 2$. J Biol Chem 271:7440-7444

46. Zha H, Reed JC (1997) Heterodimerization-independent functions of cell death regulatory proteins $\mathrm{Bax}$ and $\mathrm{Bcl}-2$ in yeast and mammalian cells. J Biol Chem 272:31482-31488

47. Hirotani M, Zhang Y, Fujita N, Naito M, Tsuruo T (1999) $\mathrm{NH} 2$-terminal $\mathrm{BH} 4$ domain of $\mathrm{Bcl}-2$ is functional for heterodimerization with Bax and inhibition of apoptosis. J Biol Chem 274:20415-20420

48. Minn AJ, Kettlun CS, Liang H, Kelekar A, Heiden MGV, Chang BS, Fesik SW, Fill M, Thompson CB (1999) Bcl-xL regulates apoptosis by heterodimerization-dependent and -independent mechanisms. Embo J 18:632-643

49. Dlugosz PJ, Billen LP, Annis MG, Zhu W, Zhang Z, Lin J, Leber B, Andrews DW (2006) Bcl-2 changes conformation to inhibit Bax oligomerization. Embo J 25:2287-2296

50. Jeong SY, Gaume B, Lee YJ, Hsu YT, Ryu SW, Yoon SH, Youle RJ (2004) Bcl-x(L) sequesters its C-terminal membrane anchor in soluble, cytosolic homodimers. Embo J 23:21462155

51. Degterev A, Lugovskoy A, Cardone M, Mulley B, Wagner G, Mitchison T, Yuan J (2001) Identification of small-molecule inhibitors of interaction between the BH3 domain and Bcl-xL. Nat Cell Biol 3:173-182

52. Vieira HL, Boya P, Cohen I, El Hamel C, Haouzi D, Druillenec S, Belzacq AS, Brenner C, Roques B, Kroemer G (2002) Cell permeable BH3-peptides overcome the cytoprotective effect of Bcl-2 and Bcl-X(L). Oncogene 21:1963-1977

53. Yin H, Lee GI, Sedey KA, Rodriguez JM, Wang HG, Sebti SM, Hamilton AD (2005) Terephthalamide derivatives as mimetics of helical peptides: disruption of the $\mathrm{Bcl}-\mathrm{x}(\mathrm{L}) / \mathrm{Bak}$ interaction. J Am Chem Soc 127:5463-5468

54. Oltersdorf T, Elmore SW, Shoemaker AR, Armstrong RC, Augeri DJ, Belli BA, Bruncko M, Deckwerth TL, Dinges J, Hajduk PJ, Joseph MK, Kitada S, Korsmeyer SJ, Kunzer AR, Letai A, Li C, Mitten MJ, Nettesheim DG, Ng S, Nimmer PM, O'Connor JM, Oleksijew A, Petros AM, Reed JC, Shen W, Tahir SK, Thompson CB, Tomaselli KJ, Wang B, Wendt MD, Zhang H, Fesik SW, Rosenberg SH (2005) An inhibitor of Bcl-2 family proteins induces regression of solid tumours. Nature 435:677681

55. Degterev A, Lugovskoy A, Cardone M, Mulley B, Wagner G, Mitchison T, Yuan J (2001) Identification of small-molecule inhibitors of interaction between the $\mathrm{BH} 3$ domain and Bcl-xL. Nat Cell Biol 3:173-182

56. D'Alessio M, De Nicola M, Coppola S, Gualandi G, Pugliese L, Cerella C, Cristofanon S, Civitareale P, Ciriolo MR, Bergamaschi A, Magrini A, Ghibelli L (2005) Oxidative Bax dimerization promotes its translocation to mitochondria independently of apoptosis. Faseb J 19:1504-1506 